\title{
Implementação de comitês de bioética em hospitais universitários brasileiros: dificuldades e viabilidades
}

Suely Marinho ${ }^{1}$, Alexandre Costa ${ }^{2}$, Marisa Palácios ${ }^{3}$, Sérgio Rego ${ }^{4}$

\section{Resumo}

Este artigo objetiva apresentar e discutir o processo de implantação e funcionamento de comitês de bioética clínica no complexo hospitalar de uma universidade pública brasileira, na qual quatro de seus hospitais são dotados dessas comissões, em diferentes etapas de estruturação: um hospital geral, um psiquiátrico, um hospital infantil e uma maternidade. Para isso, são discutidos meios, dificuldades e viabilidade para sua implantação, além de recomendações práticas em forma de etapas, com relatos de experiências no Brasil e no mundo. Com o presente texto busca-se contribuir para a reflexão bioética sobre os crescentes desafios éticos decorrentes dos desenvolvimentos científicos da biomedicina, que se materializam na atenção em saúde nas instituições. Busca-se, ainda, fomentar um importante passo para o desenvolvimento de comitês de bioética em nosso país e a apreciação crítica desse importante dispositivo de deliberação ética.

Palavras-chave: Bioética. Comitês de ética clínica. Ética institucional.

\section{Resumen}

Implementación de comités de bioética en hospitales universitarios brasileños: dificultades y viabilidades El objetivo de este artículo es presentar y discutir el proceso de implantación y funcionamiento de comités de bioética clínica en el complejo hospitalario de una universidad pública brasileña, con cuatro de sus hospitales poseyendo dichos comités, en diferentes etapas de estructuración: un hospital general, uno psiquiátrico, un hospital infantil y un pabellón de maternidad. Para esto, son discutidos medios, dificultades y viabilidad para su implantación, además de recomendaciones prácticas en forma de etapas, con relatos de experiencias en Brasil y en el mundo. Con el presente relato se busca contribuir a la reflexión bioética sobre los crecientes desafíos éticos derivados de los desarrollos científicos de la biomedicina, que se materializan en la atención a la salud en las instituciones. Se busca también fomentar un importante capítulo del campo que es el futuro desarrollo de comités de bioética en nuestro país y la apreciación crítica de este importante dispositivo de deliberación ética.

Palabras-clave: Bioética. Comités de ética clínica. Ética institucional.

\begin{abstract}
Implementation of bioethics committees in Brazilian university hospitals: difficulties and viability

The objective of this article is to present and discuss the process of implementation and functioning of clinical bioethics committees in the hospital complex of a Brazilian public university, with four of its hospitals having these commissions in different structuring stages: a general hospital; a psychiatric hospital; a children's hospital and a maternity ward. For this, alternatives, difficulties, as well as the viability, are discussed for their implementation, in addition to practical recommendations in the form of steps, with accounts of experiences in Brazil and around the world. The aim of this report is to contribute to the bioethical reflection regarding the increasing ethical challenges arising from scientific developments in biomedicine. It is also the aim of this report to encourage the future development of bioethics committees in our country and the critical appraisal of this important item of ethical deliberation.
\end{abstract}

Key words: Bioethics. Clinical ethics committee. Institutional ethics.

1. Doutora sumarinho10@gmail.com 2. Doutor alexandrecostaleal@hotmail.com 3. Doutora marisa.palacios@gmail.com-Universidade Federal do Rio de Janeiro, Rio de Janeiro/RJ, Brasil 4. Doutor starego@gmail.com - Escola Nacional de Saúde Pública, Fiocruz, Rio de Janeiro/RJ, Brasil.

Suely Oliveira Marinho - Instituto de Estudos em Saúde Coletiva (lesc/UFRJ)/PPGBIOS. Avenida Horácio Macedo, s/nº - próximo à Prefeitura Universitária da UFRJ, Ilha do Fundão - Cidade Universitária CEP 21.941-598. Rio de Janeiro/RJ, Brasil. 
O objetivo deste artigo é apresentar e discutir o processo de implantação e funcionamento de comitês de bioética clínica no complexo hospitalar de uma universidade pública brasileira. Composta por diversas unidades de saúde, quatro de seus hospitais possuem essas comissões, em diferentes etapas de estruturação: um hospital geral, um psiquiátrico, um hospital infantil e uma maternidade.

Os comitês de bioética clínica ou hospitalar, como são denominados no Brasil, foram concebidos no período de 1960 a 1970, nos Estados Unidos da América (EUA), como estrutura que tinha por função apreciar a pertinência de decisões referentes ao tratamento de pacientes individuais e, em particular, de determinar quando era adequado interromper $o$ tratamento de manutenção artificial da vida ${ }^{1}$, sem no entanto tomar a decisão final. Inicialmente destinados a auxiliar a tomada de decisão sobre o fim de vida, avaliando se o tratamento em vista dos avanços técnicos era adequado, razoável ou "ordinário", numa análise mais ética do que técnica, como originalmente proposto pela pediatra Karen Teel, os comitês ampliaram-se. Recentemente, suas atividades também se voltaram para o desenvolvimento de políticas institucionais e ações de educação relativas à ética clínica de unidades de saúde em todo o mundo ${ }^{1,2}$.

As ações descritas neste trabalho fazem parte de projeto de extensão na área da bioética clínica, voltado à implantação e ao desenvolvimento de comissões de bioética no complexo de hospitais da universidade em referência. O projeto é realizado no âmbito do Programa de pós-graduação em associação de instituições de ensino superior em Bioética, Ética Aplicada e Saúde Coletiva, promovido em parceria pela Universidade Federal do Rio de Janeiro (UFRJ), Fundação Oswaldo Cruz (Fiocruz), Universidade do Estado do Rio de Janeiro (Uerj) e Universidade Federal Fluminense (UFF), em nível de pós-doutorado (PNPD-Capes), e pelo Laboratório de Bioética Clínica da área de Bioética do Instituto de Estudos em Saúde Coletiva (IESC) da UFRJ. O relato está baseado nas atividades dos membros da equipe que implantaram e participam das reuniões de todos os comitês, com vistas a promover seu desenvolvimento e institucionalização.

$\mathrm{O}$ trabalho está dividido em três partes. $\mathrm{Na}$ primeira são apresentadas, com base na literatura científica nacional e internacional, a definição, as origens, funções e composição dos comitês de bioética. Na segunda, são discutidos os meios, dificuldades e viabilidade para sua implantação, com relatos de experiências no Brasil e no mundo. Na terceira, é apresentado e discutido o processo de implantação e desenvolvimento dos comitês com base na experiência do projeto em referência. Com a apresentação do presente relato busca-se contribuir para a reflexão bioética sobre os crescentes desafios éticos decorrentes dos desenvolvimentos científicos da biomedicina, que se materializam na atenção em saúde nas instituições. Busca-se ainda fomentar um importante capítulo do campo: desenvolvimento futuro de comitês de bioética em nosso país e a apreciação crítica desse importante dispositivo de deliberação ética.

\section{Comitês de bioética: origens, funções e carac- terísticas}

Os anos 60 e 70 foram marcados por intensos debates públicos e críticas voltadas não só para as problemáticas em torno da ética em pesquisa e os abusos cometidos, mas também para as questões éticas relacionadas à assistência em saúde, principalmente pela intensa utilização de novas tecnologias. A estruturação de comitês de bioética clínica e de outras instâncias de análise e deliberação ética, como os de ética em pesquisa, em vários países, em especial nos EUA, constituiu uma das expressões de tais críticas, contribuindo tanto para a institucionalização da bioética na área da saúde como para sua consolidação acadêmica ${ }^{2}$.

Assim, as origens dos comitês de bioética remontam aos EUA, com destaque aos casos judiciais paradigmáticos que mobilizaram a opinião pública em razão, por um lado, do descompasso entre os avanços na saúde e os critérios de escolha dos beneficiários, por outro, em decorrência dos conflitos em torno das condutas indicadas em casos clínicos limite. Havia o questionamento sobre as deficiências do sistema e das dificuldades de a equipe médica solucionar situações clínicas de conflitos éticos (Comitê de Seattle/EUA, Quinlan, Baby Doe $)^{2-7}$. Esses casos tiveram em comum divergências entre família, responsáveis e equipe de saúde sobre as decisões em torno dos procedimentos médicos, que foram mediadas pelo recurso judicial.

A ideia de uma estrutura que pudesse deliberar sobre situações de conflito nos cuidados individuais de pacientes ganhou impulso com a recomendação judicial desses casos, mas não se desenvolveu plenamente: em 1983, apenas $1 \%$ dos hospitais possuíam comissões de ética em seus quadros, conforme estudo da Comissão Presidencial para o Estudo de Problemas Éticos na Medicina e na Pesquisa Biomédica e Comportamental ${ }^{1}$. Somente ao fim da década de 80 sua formalização tornou-se realidade, 
generalizando-se em quase todos os hospitais estadunidenses, com o apoio de organizações como a Associação Médica Americana (AMA). Por fim, com o Manual de Acreditação para Hospitais, de 1992, da Joint Commission on Accreditation of Healthcare Organizations, ficou estabelecida, como critério de qualidade e condição de credenciamento, a existência de serviços de consultoria ética no processo assistencial, modificando o panorama de $1 \%$ de comitês em hospitais, em 1983, para 60\% em 1989 e 93\% próximo ao ano $2000^{8}$.

Considera-se também um significativo avanço para a implementação dos comitês em outros países a aprovação, pelos países-membros da Unesco, em 2005, da Declaração Universal sobre Bioética e Direitos Humanos, que recomenda a criação e apoio aos comitês, tanto para avaliar os problemas éticos, jurídicos, científicos e sociais relevantes no que se refere aos projetos de investigação envolvendo seres humanos como para dar pareceres sobre os problemas éticos em contextos clínicos ${ }^{9}$.

Nesse novo contexto, verifica-se aumento da demanda por formação no campo da bioética e maior penetração social da ética clínica. Os estudos mostram maior aceitação pelos profissionais de saúde das discussões sobre ética ${ }^{10}$, em especial aquelas que envolvem os casos clínicos de maior complexidade. Se numa primeira etapa o desenvolvimento de comitês de bioética originou-se dos desafios trazidos pelo progresso técnico da medicina com seus novos padrões de tratamento, num contexto de diversidade cultural ${ }^{4,11}$, atualmente 0 movimento crescente de judicialização da saúde e a maior consciência dos direitos têm constituído força motriz para tal abertura. Para Vasconcelos ${ }^{12}$, a judicialização excessiva, como árbitro da relação dos usuários com o sistema de saúde, tem se mostrado um equívoco na busca por maior simetria nessa relação. Na visão da autora, o acesso à justiça, principalmente em vista de grandes desigualdades sociais, pode significar ampliação da cidadania e redução da vulnerabilidade, contudo, tornando-se regra, pode significar a substituição de uma relação de atenção ao cuidado por uma de beligerância, produzindo-se a assim chamada "medicina defensiva" ${ }^{12}$.

Nas instituições de saúde, em síntese, as discussões mais amplas sobre os casos clínicos de maior dificuldade do ponto de vista da tomada de decisão em situações de conflito moral têm sido realizadas nessa nova estrutura que são os comitês de bioética. Trata-se de instância na qual participam profissionais de saúde e de outras áreas, como teólogos, juristas e filósofos, bem como representantes dos usuários e da comunidade, sendo multiprofissional e multidisciplinar ${ }^{2,3}$. Sua proposta é constituir-se como espaço plural e dialógico, que valorize todos os atores na busca por soluções de conflitos no cenário da saúde.

Embora a proposta seja de horizontalização das relações de forças presentes, Singer e colegas ${ }^{13}$ chamam a atenção para o perigo de seus consultores ultrapassarem a função de recomendar cursos de ação e assumirem o processo de tomada de decisão, bem como dos profissionais seguirem as recomendações sem ponderá-las, tendo em vista o medo de ações judiciais.

Apesar dos potenciais perigos, os comitês de bioética têm sido defendidos por diversos autores como ferramenta útil capaz de facilitar a tomada de decisões em ambientes institucionais pela ampliação da discussão entre aqueles que enfrentam desafios éticos referentes ao cuidado de um paciente ${ }^{14}$. Singer e colegas ${ }^{13}$, em 1990, afirmaram que os serviços de consulta se tornariam ainda mais importantes nos próximos anos com o progresso tecnológico, o pluralismo moral e intervenções legais, dificultando ainda mais a tarefa de tomada de decisão. Ao lado desses, outros aspectos da prática assistencial justificam a criação dos comitês de bioética, como o reconhecimento da autonomia do paciente e as convicções religiosas de grupos, além da necessidade de discutir eticamente a alocação de recursos disponíveis ${ }^{15}$.

Para que a consulta ética cumpra com os objetivos propostos são preconizadas abordagens diferentes: os comitês de bioética, propriamente, e os consultores individuais. A American Society for Bioethics and Humanities (ASBH) propõe modelo misto ou formado por pequenas equipes, sendo o modelo de comitê o mais frequente ${ }^{8}$. Esses modelos atingem seus objetivos mediante três atividades: 1) coordenação de programas de educação em ética, inclusive a formação dos próprios membros do comitê; 2) auxílio no desenvolvimento de políticas institucionais que definem o posicionamento ético da unidade de saúde ante determinadas situações e; 3) consultas em casos individuais, nos quais se busca a identificação das questões morais e possíveis conflitos que o caso suscite ${ }^{4,5,13}$.

Singer e colegas ${ }^{13}$ apresentam quatro modelos de consulta em ética clínica, com suas vantagens e desvantagens:

a) modelo puro de comitê - o solicitante, que pode ser um familiar ou o profissional, apresenta o caso à comissão e esta discute e faz suas recomendações; 
b) membro do comitê como consultor - o pedido é feito ao comitê, que designa um de seus membros para analisar o caso. Os resultados da análise são apresentados ao comitê, que os discute e faz recomendações;

c) comitê de análise pós fato - o pedido é feito diretamente ao consultor, que analisa e faz as recomendações. $\mathrm{O}$ caso é posteriormente analisado por um comitê;

d) modelo puro de consulta - o pedido é feito diretamente ao consultor, que analisa e faz recomendações, sem que estas sejam analisadas posteriormente pelo comitê.

Em termos de vantagens, o modelo puro de comitê propicia maior consenso institucional e apoio às recomendações. Por sua vez, o modelo puro de consulta propicia resposta mais rápida e maior eficiência. Os modelos que combinam as estratégias de comitê e o consultor individual garantem discussão mais ampla e maior controle das decisões ${ }^{13}$. De acordo com Ribas-Ribas ${ }^{8}$, autores como La Puma e Toulmin, Siegler, bem como o grupo de trabalho da ASBH, concluíram que não deve haver escolha exclusiva de qualquer um desses modelos, pois cada um se aplica bem a situações distintas.

Francisconi, Goldim e Lopes ${ }^{5}$ sintetizam a discussão ao afirmarem que um comitê de bioética possui tríplice função: educativa, consultiva e normativa, com avaliação e discussão de questões e dilemas morais com origem na prática e procedimentos no âmbito da instituição, com vistas ao meIhoramento do atendimento ofertado ${ }^{4}$. Destaca-se que o comitê de bioética difere de outras comissões de ética que igualmente atuam nas instituições de saúde, como as Comissões de Ética e Deontologia Médica e as de Enfermagem, voltadas para a observação do cumprimento dos deveres profissionais de médicos e enfermeiros. Além disso, tem-se as Comissões de Ética em Pesquisa, que visam avaliar a adequação ética dos projetos de pesquisa envolvendo seres humanos ${ }^{4}$.

Implantação de comitês de bioética: experiências no Brasil e no mundo

Nesta seção serão apresentadas e discutidas algumas medidas concretas utilizadas para a implantação e desenvolvimento de comitês de bioética, os problemas mais comumente envolvidos, bem como breve apreciação de sua implementação em alguns países e no Brasil. Estudo realizado por Vollmann em $2010{ }^{16}$ buscou demonstrar a importância do processo de implantação dos comitês, que, na prática, tem sido um processo complexo e mesmo difícil, em razão de conflitos de interesses e de resistência para mudanças.

Estudos sobre a implantação e funcionamento de comitês de bioética hospitalar têm sido publicados também na Europa e já apresentam tempo de existência suficiente para permitir a avaliação dessas experiências ${ }^{17}$. Na prática de países europeus como Alemanha, Itália, Holanda e nos chamados países "de transição", como Geórgia, Croácia e Bulgária, as estruturas dos comitês de bioética, denominados nesses países de Clinical Ethics Consultation, foram comumente implementadas por iniciativa oficial do Estado ou por iniciativas institucionais que partem da gestão no âmbito de processos de certificação de instituições de saúde ${ }^{17,18}$. Exemplifica o processo a criação de comitês de ética na Geórgia, que ganhou impulso em 2003, determinada pela legislação nacional, com recomendação do Conselho Nacional de Bioética, criado em $2000{ }^{19}$. Na Holanda ${ }^{18}$, país com maior tradição nesse campo, em 2005 o Centro de Ética em Cuidado de Saúde aconselhou o governo a dar mais atenção à deliberação moral de forma estrutural entre os profissionais de saúde, numa proposta de relação permanente entre deliberação e política institucional.

Essas estratégias de implantação e implementação dos comitês de "cima para baixo" são definidas por Vollmann ${ }^{16}$ e Schildmann ${ }^{14}$ como abordagem top-down. Significa que as iniciativas estruturais são realizadas pela gestão, com garantia de aportes materiais e de infraestrutura, o que pode garantir do ponto de vista institucional a efetiva criação dessa inovação pelo envolvimento de quem está em posição de maior poder de decisão e pela capacidade de maior regulação ${ }^{19}$.

Uma outra estratégia de implementação é denominada bottom-up, com origem da iniciativa de "baixo para cima", isto é, parte de funcionários que veem a necessidade de discussão regular sobre questões éticas que surgem no trabalho diário. Essa abordagem tem a vantagem de partir do próprio ambiente de trabalho, aspecto crucial para a aceitação e a vitalidade dos comitês no contexto institucional ${ }^{16}$.

Na Argentina ${ }^{20}$, pioneira da América Latina na criação de comitês de ética, houve dois momentos na estruturação dessas instâncias. 0 primeiro, mais lento, resultou na consolidação de muitos comitês e em sua transformação em centros de referência cujas estruturas, no segundo momento, obedeceram ao marco regulatório legal sancionado na década de 90. Apesar da regulação pela Lei Nacional de Criação 
de Comitês de Ética de $1996^{20}$, seu crescimento foi desordenado, sem o apoio do Ministério da Saúde e num contexto de privatização dos serviços essenciais.

Conforme Luna e Bertomeu ${ }^{20}$, o surgimento dos comitês no país deveu-se bem mais à pressão legal do que ao reconhecimento de uma necessidade. Isso gerou inúmeros problemas, desde os relacionados à aceitabilidade pelos profissionais até a falta de diferenciação quanto a funções, diferentes poderes e a formação requerida entre comitês de ética clínica e comitês de ética em pesquisa no texto da lei. Esse processo de criação foi exceção somente para os hospitais, que, em razão dos dilemas éticos próprios de sua especialização, como um hospital infantil e outro de cuidados de pacientes com aids, criaram seus comitês de ética antes ou paralelamente às legislações.

No Brasil, diferentemente, a estruturação das comissões de bioética ocorre por iniciativa da unidade de saúde e não por legislação nacional ou mesmo diretriz que verse sobre sua criação. Ainda há pouca tradição na constituição de tais estruturas nas unidades de saúde, bem como na formação e treinamento dos profissionais em questões éticas. Contudo, a partir dos anos 90 verifica-se modificação nesse panorama e as primeiras comissões de bioética são implementadas, com destaque às experiências do Hospital das Clínicas de Porto Alegre (UFRS), com o Programa de Apoio aos Problemas de Bioética, em 1993; do Hospital São Lucas (PUCRS), em $1997^{4-6}$; do Hospital das Clínicas de São Paulo (USP) - CoBi/HCFMUSP, em $1996{ }^{21}$; do Instituto Nacional de Câncer, no Rio de Janeiro (ConBio), instituído em 1999 22; e do HUCFF-UFRJ, em $2003{ }^{23}$.

Apesar da alteração do quadro pela criação dessas comissões de bioética, ainda são poucos os relatos sobre a experiência e história dos comitês no Brasil. Entretanto, têm ocorrido mudanças e em 2011 e 2012 pelo menos dois comitês divulgaram suas experiências: o primeiro foi o relato de caso sobre a criação do Comitê de Bioética do Hospital Infantil Joana Gusmão, em Florianópolis/SC, com início das atividades educativas em 2010 e das atividades consultivas em 2011. A criação ocorreu após sensibilização dos profissionais (corpo clínico e funcionários de diversos serviços) sobre funções, composição e formas de funcionamento; estabelecimento de critérios para escolha dos membros e constituição de um núcleo organizador ${ }^{15}$. O segundo trabalho ${ }^{24}$ foi a divulgação de pesquisa que buscou averiguar os principais êxitos e problemas dos três primeiros anos de funcionamento (2007-2009) do Comitê de Bioética do Hospital Universitário San- ta Teresinha, da Universidade do Oeste de Santa Catarina (Joaçaba/SC) - considerando serem ainda raros os estudos de avaliação, este último modifica tal perspectiva no Brasil.

Retomando a questão das abordagens topdown e bottom-up, ressalta-se que essas diferentes realidades da instituição devem convergir para que se obtenha a implementação adequada e o desenvolvimento efetivo do comitê. Vollmann ${ }^{16}$ propõe um modelo com caráter de recomendação para a implantação e o desenvolvimento dos comitês de bioética, que do ponto de vista prático tem se revelado muito útil àqueles profissionais que aceitam o desafio de implementá-los em suas instituições.

Um primeiro aspecto a observar é que, para a implementação exitosa das comissões, as funções, as atribuições, a composição e os limites de ação precisam ser claramente definidos. Um segundo aspecto é o enfrentamento das objeções que surgem na prática por parte dos profissionais, com destaque à alegação de falta de tempo; da visão da comissão como algo imposto pela administração, principalmente se ordenada pela gestão de maneira muito rápida em razão de processos de certificação; à ideia de interferência na relação de confiança médico-paciente ${ }^{8,16}$ ou ainda de aumento da carga burocrática ${ }^{16,20}$.

Nesse sentido, argumenta-se que a reflexão ética e a discussão ampla promovem o aumento no repertório de ação e maior capacidade de análise dos profissionais em razão do compartilhamento das situações complexas, tornando o trabalho e as ações mais eficazes e resolutivas. Além disso, em razão do compartilhamento das responsabilidades, a própria equipe pode ser fator protetor para o profissional contra o adoecimento e os problemas de burnout em razão das sobrecargas oriundas da natureza do trabalho. Isso acaba por trazer melhoramentos para um aspecto da instituição muito negligenciado que, cada vez mais, é apontado como fundamental na prática clínica em estudos e experiências: a comunicação, seja em situações clínicas limite entre equipe, paciente e sua família, seja entre os profissionais ${ }^{25}$, promovendo melhorias no atendimento ofertado e no ambiente de trabalho.

Em seu estudo, Vollmann ${ }^{16}$ apresenta algumas recomendações práticas, sob a forma de seis etapas, para ajudar na fundação e implementação dos comitês. Como recomendações, são passíveis de modificações e adaptações para a realidade, história e objetivos das diferentes instituições e comissões.

Em linhas gerais, a etapa 1 do programa de implantação ${ }^{16}$ está voltada para a "administração" 
da instituição - entendimento do contexto de surgimento da demanda e se há um pedido formal de fundação do comitê. No trabalho de fundação é central a definição das regras e objetivos do comitê, bem como de horas trabalhadas na carga horária do funcionário, de recursos materiais e de infraestrutura que viabilizem sua implantação.

A etapa 2 volta-se para as atividades iniciais de um "coordenador" que busca na instituição os profissionais que se interessam em participar do comitê. Isso ocorre por meio de divulgação interna dos objetivos, fundação de um grupo de trabalho com discussão de temas de interesse da comunidade, inclusão de estruturas existentes na instituição, como membros do comitê de ética profissional, comitê de ética em pesquisa etc. A etapa 3 volta-se para o "grupo de trabalho", com a estruturação da comissão com membros permanentes e de diferentes grupos profissionais da instituição, além de membros externos. É importante estabelecer a periodicidade das reuniões, para favorecer a inclusão da comissão no fluxo organizacional, além de iniciar a reflexão sobre o método de discussão com base nas referências conceituais e de princípios da bioética, respeitando-se, contudo, o estilo de cada estrutura.

A etapa 4 - "comitê de bioética clínica" - está ligada à fundação propriamente dita, cuja estratégia pode ser associá-la a um evento da instituição em que possam ser apresentados e discutidos temas práticos e de interesse local. A partir da existência institucional do comitê, por meio de portarias de nomeação dos membros, é importante estabelecer prazo para a renovação dos mesmos, que pode ser de dois a três anos, com um número viável de participantes. Além disso, é importante elaborar estatutos que regulem procedimentos e regras, mas não em muitos detalhes, para não restringir o trabalho diário futuramente. Após a fundação, chegase à etapa 5 , na qual acontece o desenvolvimento do trabalho conceitual e comunicativo. Isso significa promover palestras regulares sobre temas sensíveis relacionados à prática dos profissionais, estimular a discussão e mesmo formação e capacitação que orientem a tomada de decisão dos profissionais, baseada na reflexão ética e na argumentação, aspectos ainda ausentes na educação acadêmica.

A etapa 6 implica especialização do serviço, com o desenvolvimento de consulta ética por especialidades clínicas, pois os problemas numa comissão podem variar muito de uma área especializada para outra. Por essa razão, pode ser útil adaptar a configuração e o procedimento de trabalho para as especialidades como psiquiatria, pediatria, medi- cina intensiva, com questões sobre início e fim de vida, cuidados paliativos etc. Outras recomendações importantes dessa etapa são a construção de rede com outras comissões; garantia de sigilo (discussão sobre segurança e confidencialidade); pesquisa científica sobre comitês de bioética clínica e cooperação internacional para o intercâmbio de experiências ${ }^{16}$.

\section{A criação de comitês de bioética em uma universidade pública brasileira}

Esta seção apresenta o relato de caso referente à implantação de comitês de bioética em uma universidade pública brasileira, em quatro de suas unidades de saúde: um hospital geral de adulto, um hospital infantil, um de psiquiatria e uma maternidade, com características bem distintas e em diferentes fases de desenvolvimento. A implantação dos comitês de bioética nessas unidades ocorreu no período de 2010 a 2012, à exceção do hospital geral de adulto, que é anterior. Para as demais unidades, as etapas propostas no projeto de implantação foram:

1. Procura da direção da unidade para exposição do projeto de implantação do comitê;

2. Exposição do projeto ao corpo social da unidade (geralmente em atividades de centros de estudos ou instâncias similares), com tempo para discussão, objetivando sensibilizar o grupo sobre a importância, funções e características dos comitês de bioética;

3. Identificação, em colaboração com a direção e profissionais interessados, dos membros permanentes da comissão (é recomendado aproximadamente 20 membros, com caráter multidisciplinar, incluindo-se pelo menos um participante que não pertença à área da saúde);

4. Estabelecimento, pela unidade, da periodicidade das reuniões (com exceção do hospital infantil, no qual é quinzenal, as demais unidades optaram por reuniões mensais);

5. Estabelecimento da dinâmica das reuniões, com tempo de duração máxima de 60 a 90 minutos.

A dinâmica proposta para as reuniões foi organizada da seguinte forma:

- notícias relevantes dos últimos casos;

- relato do caso pelo profissional solicitante do parecer à comissão e aos demais profissionais diretamente envolvidos no cuidado, com exposição dos aspectos "técnicos" numa perspectiva interdisciplinar (quadro clínico, situação institucional, 
jurídica, social, cultural e emocional do paciente e familiares);

- identificação das questões morais e possíveis conflitos que o caso suscita. Discussão com ampliação das informações e identificação dos cursos de ação possíveis para dar subsídio à tomada de decisão ética pela equipe responsável. Elaboração e apresentação de argumentos;

- registro, em livro, das reuniões.

\section{Hospital geral de adultos}

A fundação desse comitê ocorreu em julho de 2003 e buscou, inicialmente, congregar os diversos serviços do hospital. Ao longo dos anos, houve períodos em que a comissão funcionou de maneira pouco sistemática, com reuniões esporádicas. Apesar disso, apresentou desdobramentos importantes na dinâmica da instituição. Além de atribuições como fomentar o debate bioético internamente, visando colaborar na formação de estudantes de graduação e pós-graduação e profissionais de saúde, a comissão tinha ainda um conjunto de atividades pedagógicas, tais como: 1) participação no curso anual de formação de residentes; 2 ) inclusão de disciplina obrigatória de bioética nos cursos de pós-graduação em medicina da universidade; 3 ) criação das disciplinas de bioética e bioética clínica - eletivas da graduação em medicina.

A experiência com os residentes (oficina de bioética), registrada em artigo de 2009 de autoria dos membros da comissão à época ${ }^{23}$, ocorreu no segundo semestre de 2003 - fruto de parceria com a Comissão de Residência Médica (Coreme) do hospital - e objetivava fomentar o debate sobre os aspectos éticos e bioéticos da prática profissional em saúde.

Em 2011, já no âmbito do projeto em referência, foi retomado o contato com alguns dos membros, em especial o Serviço de Psiquiatria e Psicologia Médica e o coordenador da comissão. Ficou pactuada a retomada de seu funcionamento regular. Nesse ano, as reuniões passaram a ocorrer a cada seis meses, com a presença dos membros da comissão, médicos residentes e estudantes da graduação de medicina. Em 2012 e 2013, foram mensalmente retomadas as reuniões.

As discussões nessa comissão giraram em torno de temas variados, com destaque para: questões relativas à terminalidade da vida ("eutanásia", "distanásia", "morte digna", "morte natural", Resolução CFM 1.805/06, que regula a chamada "ortotanásia", tomada de decisão nos momentos finais da vida do paciente, ausência de serviços de cuidados paliati- vos); confidencialidade e a crescente judicialização da saúde. E, ainda, o problema de "rede" do sistema de saúde; falta de integração entre as equipes, deixando-se o paciente sem referência, com descontinuidade do tratamento; dificuldade de tratar pacientes Testemunhas de Jeová e outras questões. $\mathrm{Na}$ página eletrônica da comissão há informação acerca das discussões éticas sobre esses aspectos da prática assistencial ${ }^{26}$.

Nessa retomada, uma característica que tem marcado a comissão do hospital de adultos é o fato de estar sendo implementada de forma mais próxima à estratégia bottom-up, isto é, com a iniciativa de profissionais ligados ao ensino da medicina no hospital, mas sem a participação efetiva da Direção. Também há pouca participação de estudantes de outras áreas da saúde, além da medicina. Esses aspectos se apresentam como desafios para melhor análise futura.

\section{Hospital infantil}

No hospital infantil, a implantação do comitê de bioética ocorreu em 2011, funcionando com reuniões quinzenais, a pedido da própria instituição por haver elevado número de casos de conflitos morais.

As reuniões da comissão contam com frequente participação de seus membros e da administração do hospital, com intensa atividade de discussão de casos. A discussão gira em torno de temas bastante diversificados, como respeito à autonomia do paciente menor; autonomia dos pais e os melhores interesses da criança; alta de paciente com doença crônica muito grave, a pedido da família, o que gera incertezas na equipe assistencial. Um tema que se tornou emergente no início dos trabalhos da comissão foi o da prática de "discriminação positiva" voltada ao paciente quando contaminado por bactérias multirresistentes: é razoável segregá-lo? O que isso significa do ponto de vista do tratamento? E do ponto de vista moral?

Foram realizados três encontros extraordinários com os profissionais para ampliar a discussão relativa a essa temática - do que resultou uma proposta de modificação da política institucional perante essa situação.

A proposta de mudança ocorreu por intermédio da Comissão de Controle de Infecção Hospitalar $(\mathrm{CClH})$, que não via necessidade de segregação absoluta desses pacientes contaminados em relação aos demais, trazendo como argumentos uma série de esclarecimentos técnicos, como os meios de contaminação e a ineficácia da segregação como forma 
de prevenir novas infecções. Tais esclarecimentos técnicos, no entanto, ocorreram após um processo inicial de manifestação de todos os profissionais envolvidos e detecção dos problemas e das formas de entendimento da questão, em que cada um pôde expressar opiniões, argumentos e propostas sobre a prática ou não de discriminação.

Nesse caso, é importante ressaltar, a discussão ética ultrapassou a questão da moralidade da discriminação positiva para propor um modelo de resolução de conflito centrado na participação de todos os envolvidos, para o encontro da melhor solução.

\section{Hospital psiquiátrico}

No hospital em referência, o processo de implantação ocorreu em março de 2012, com a exposição do projeto à Direção e posterior palestra para a comunidade no Centro de Estudos. Em maio daquele ano, foi realizado o primeiro encontro do comitê de bioética na instituição. A Portaria 4.125 da Direção, de 28 de maio de 2012, nomeou os membros para compor o comitê no exercício de 2012 a 2015.

Nesse comitê, o tema emergente tem sido o dos limites da autonomia do paciente com transtornos mentais e as dificuldades para prover o cuidado à medida que há interdependência de aspectos legais, sociais e de saúde desses pacientes, com o surgimento de dispositivos jurídicos, como interdições totais, internações compulsórias por determinação judicial. Outro tema sensível é o das dificuldades da assistência ao enfermo dependente químico, clientela que tem aumentado em número, nos últimos anos, no hospital. Com base nas questões abordadas, foi planejada pela equipe do projeto uma palestra para o hospital, com discussão sobre o tema da autonomia de assistidos com transtornos mentais.

O absenteísmo tem sido uma das problemáticas do comitê nessa instituição. Alguns participantes alegam carga horária pesada e falta de tempo para se envolver com mais uma atividade, o que confirma dados da literatura que apontam maior dificuldade para a eficaz implementação dessa estrutura em hospitais psiquiátricos. As razões apontadas seriam maior competência desses profissionais no trabalho em equipe, na comunicação e no manejo de situações de conflito no cotidiano do trabalho, levando-os a desconsiderar a necessidade de consulta ética ${ }^{27}$, devendo tal aspecto ser objeto de maior aprofundamento na comissão em referência.

\section{Maternidade}

Na maternidade, o processo incluiu a apresentação do projeto à Direção, em fins de 2012, e à comunidade do hospital, no Centro de Estudos, no início de 2013. Após essas atividades, a unidade se organizou internamente e convocou membros que pudessem contribuir para elaborar a portaria de criação da comissão. Após esses trâmites organizacionais, o calendário foi estabelecido e no primeiro encontro foi discutida a questão do sigilo e da confidencialidade na assistência materno-infantil, especialmente nos casos de contaminação pelo HIV de mãe para filho.

Outras questões discutidas foram o problema da alta à revelia, bem como as dificuldades de lidar com bebês com extremo baixo peso, discussão que aponta para a fronteira entre aborto e prematuridade extrema, e demais dilemas do início da vida. Nas atividades educacionais, foi realizada uma palestra (divulgação de pesquisa) sobre o impacto para a gestante do diagnóstico de malformação fetal, de maneira a ampliar o entendimento sobre os desacordos entre família e equipe quanto à tomada de decisão.

\section{Discussão}

Este trabalho apresentou a experiência prática de implantação e desenvolvimento de comitês de bioética no complexo hospitalar de uma universidade pública brasileira. A iniciativa justifica-se, por um lado, pela presença crescente de grandes desafios éticos na prática clínica diária e, por outro, pela necessidade de ampliar a qualidade do cuidado em saúde em suas dimensões coletiva e individual mediante ferramentas de apoio, como os comitês.

Basicamente, a estratégia de implantação dos comitês no âmbito do presente projeto se ancora num plano de ação em que se busca a integração das diferentes realidades institucionais - gestão e corpo de profissionais -, de maneira que a comissão alcance existência, ganhe visibilidade e consolide sua legitimidade na unidade de saúde. Isso tem se mostrado verdadeiro desafio, quer pelas resistências a mudanças, quer pelo pouco conhecimento da especificidade dessas estruturas. Outra estratégia é a efetiva participação da equipe do projeto como membro dos comitês, viabilizando o exercício da consulta ética, bem como de ações educativas.

Os quatro comitês objetos deste trabalho têm diferentes configurações e problemáticas, bem como distintos níveis de entendimento sobre sua 
importância. Cada um está em uma fase de estruturação, com modos diversos de engajamento de seus membros, e pode-se observar que aqueles com maior grau de desenvolvimento são justamente os que conseguem articular os diferentes níveis da instituição, o que confirma os dados da literatura apresentados. Basicamente, nos comitês, se tem conseguido garantir suas três principais funções. Entretanto, nesses momentos iniciais estão mais fortemente presentes suas dimensões educativa e de consulta de casos individuais.

Embora comece a se intensificar a percepção da necessidade de comitês de bioética, há ainda número limitado de atividades nesse campo. Paralelamente, alguns estudos ${ }^{18}$ mostram que muitos profissionais de saúde começam a aceitar tais discussões, mas não têm conhecimento ético básico e habilidades para lidar com questões morais. Além disso, ainda há pouco entendimento sobre o fato de que a ética está ligada a formas cotidianas de cuidado e de que decisões técnicas são atravessadas por valores morais. Outros estudos ${ }^{10}$ mostram existir grandes diferenças entre os profissionais quanto ao que é considerado problema ético, aos modos de resolvê-los e usos de dispositivos e estratégias para esse fim - como a utilização de consulta ética, recorrer às próprias intuições e experiência ou, ainda, aos colegas mais antigos. Esses dados, por si, revelam a efetiva necessidade da criação de instâncias nas quais essas questões possam ser reconhecidas e discutidas.

Os estudos acima citados ${ }^{10,18}$, além disso, indicam que uma melhor educação ética poderá aumentar a sensibilidade a essas questões na prática e servir de base para o desenvolvimento de serviços de comitês com maior engajamento dos profissionais. Nesse sentido, é importante aumentar o ensino de bioética em todos os níveis, graduação, pósgraduação etc., incluindo-se treinamento para os membros dos comitês e maior divulgação no tecido social, sendo essa uma das vertentes organizacionais que privilegiamos em nossa experiência.

O projeto de extensão em referência, no âmbito de um programa de pós-graduação, tem propiciado o conhecimento de diferentes experiências de desenvolvimento de comitês, que ajudaram a instrumentalizar a criação de tais estruturas nos hospitais de uma importante universidade brasileira. Este artigo pretende, portanto, contribuir para o desenvolvimento de comitês em nosso país com a divulgação dos caminhos percorridos e estratégias utilizadas para sua implementação, apresentando também as dificuldades, a fim de superá-las nessa e em outras experiências.

Pode-se dizer que ainda estamos diante de um fenômeno novo, do qual muitos aspectos precisam ser desenvolvidos, como a criação de programas de treinamento em consulta ética; uma avaliação mais sistemática sobre a eficácia de suas normas éticas na prática; a adequação das teorias e dos métodos ao exercício cotidiano nas instituições e mais pesquisas no campo, visando identificar as opiniões de profissionais de saúde e administradores sobre o valor dos comitês, sua eficácia, o entendimento sobre suas características, além de maior divulgação das experiências existentes.

Como vimos, há longo caminho a ser percorrido para sua consolidação como espaço privilegiado em que as decisões são tomadas com base em um processo de reflexão e argumentação éticas, com a inclusão da complexidade que atravessa a clínica. Isso se torna especialmente importante em tempos de grande desenvolvimento tecnológico que têm transformado a vida em objeto de decisão política, revelando a necessidade de que as escoIhas sejam feitas de maneira refletida e com maior envolvimento de atores diversos, e não apenas os médicos.

Para finalizar, ressalta-se que essas contribuições para o debate têm a função de iniciar um diálogo com outras estruturas de bioética clínica de maneira a propiciar seu desenvolvimento e institucionalização no país. Para tanto, foram trazidas algumas recomendações que podem fomentar outras iniciativas, como a consideração sobre as etapas iniciais de implantação ${ }^{16}$, de maneira que os problemas não sejam subestimados e levem ao fracasso da experiência.

\section{Considerações finais}

Em sintonia com os estudos apresentados, conclui-se, em seus aspectos mais gerais, que as comissões têm constituído ferramenta útil para o encaminhamento dos casos que exigem avaliação ética, ao possibilitar abordá-los de maneira mais rica pela ampla discussão que Ihes é característica. Como vantagens, destacam-se a busca pelo protagonismo de pacientes e representantes, bem como a oferta de maior repertório de ações aos profissionais e aos gestores em saúde. Finalmente, as comissões contribuem para a identificação e condução dos dilemas éticos, tanto para os envolvidos no am- 
biente hospitalar, pacientes, sua família e profissionais, como para a sociedade em geral. Em paralelo, destaca-se que um importante aspecto a ser desen- volvido no campo é o das pesquisas de avaliação que contemplem, de maneira detalhada, o desempenho das comissões.

\section{Trabalho resultante de projeto de extensão em andamento financiado pela Capes no âmbito do Programa Nacional de Pós-Doutorado Institucional.}

\section{Referências}

1. Veakh RM. As comissões de ética hospitalar ainda têm função? Bioética. 1998;6(2):161-70.

2. Rego S, Palácios M, Siqueira-Batista R. Bioética para profissionais de saúde. Rio de Janeiro: Fiocruz; 2009.

3. Kipper, DJ, Lock JA, Gauer GJC. Comitês institucionais de bioética. In: Siqueira JE, Zoboli ELCP, Kipper DJ, organizadores. Bioética clínica. São Paulo: Gaia; 2008. p. 197-207.

4. Goldim JR, Francisconi CF. Os comitês de ética hospitalar. Bioética. 1998;6(2):149-55.

5. Franciscone CF, Goldim JR, Lopes MHI. O papel dos comitês de bioética na humanização da assistência à saúde. Bioética. 2002;10(2):147-57.

6. Goldim JR, Raymundo MM, Fernandes MS, Lopes MH, Kipper DJ, Francisconi CF. Clinical bioethics committees: a brazilian experience. J Int Bioethique. 2008;19(1-2):181-92.

7. Pessini L, Barchifontaine CP. Problemas atuais de bioética. $6^{\text {a }}$ ed. São Paulo: Loyola; 2002.

8. Ribas-Ribas S. Estudio observacional sobre los comités de ética asistencial en Cataluña: el estudio CEA-CAT (1). Estructura y funcionamento. Med Clin. 2006;126(2):60-6.

9. Organização das Nações Unidas para a Educação, a Ciência e a Cultura. Declaração Universal sobre Bioética e Direitos Humanos. [Internet]. Unesco; 2005 (acesso 5 set. 2013). Disponível: http://unesdoc.unesco.org/images/0014/001461/146180por.pdf

10. Aleksandrova S. Experience in ethical decision-making and attitudes towards ethics consultation of regional hospital physicians in Bulgaria. In: Schildmann J, Gordon, JS. Vollmann J, organizadores. Clinical ethics consultation: theories and methods, implementation, evaluation. England: Ashgate; 2010. p. 175-87.

11. Widdershoven G, Molewijk B. Philosophical foundations of clinical ethics: a hermeneutic perspective. In: Schildmann J, Gordon JS, Vollmann J, organizadores. Op. cit. p. 37-52.

12. Vasconcelos $C$. Responsabilidade médica e judicialização na relação médico-paciente. Rev. bioét. (Impr.). 2012;20(3):389-96.

13. Singer PA, Pellegrino ED, Siegler M. Ethics committees and consultants. J Clin Ethics. 1990;1(4):263-7.

14. Schildmann J. Evaluation of clinical ethics consultation: a systematic review and critical appraisal of research methods and outcome criteria. In: Schildmann J, Gordon JS, Vollmann J, organizadores. Op. cit. p. 203-15.

15. Guedert JM, Grosseman S. Comitê de bioética em hospital pediátrico: da proposta à ação. Rev. bioét. (Impr.). 2011;19(3):765-72.

16. Vollmann J. The implementation process of clinical ethics consultation: concepts, resistance, recommendations. In: Schildmann J, Gordon JS, Vollmann J, organizadores. Op. cit. p. 91-106.

17. Schildmann J, Gordon JS, Vollmann J, organizadores. Clinical ethics consultation: theories and methods, implementation, evaluation. Op. cit.

18. Stolper M, Dam S van der, Widdershoven G, Molewijk B. Clinical ethics in the Netherlands: moral case deliberation in health care organizations. In: Schildmann J, Gordon JS, Vollmann J, organizadores. Op. cit. p. 149-60.

19. Chikhladze N, Pitskhelauri N. Demands and needs in clinical ethics consultation in Georgia. In: Schildmann J, Gordon JS, Vollmann J, organizadores. Op. cit. p. 131-7.

20. Luna F, Bertomeu MJ. Comitês de ética na Argentina. Bioética. 1998;6(2):183-7.

21. Hospital das Clínicas da Faculdade de Medicina da Universidade de São Paulo. Comissão de Bioética do HCFMUSP - CoBi. [Internet]. 1997 (acesso 21 ago. 2012). Disponível: http://www. hc.fm.usp.br/index.php?option=com_content $\&$ view $=$ article $\&$ id $=242$ :comissao-bioeticacobi\&catid=23:internas

22. Instituto Nacional de Câncer. Conselho de Bioética do Inca - ConBio. [Internet]. Rio de Janeiro: Inca; 1996-2013 (acesso 17 out. 2012). Disponível: http://www.inca.gov.br/conteudo_view. asp?id $=674$

23. Zaidhaft S, Goldwasser R, Spitz L, Pimentel ML, Zornita M, Siqueira-Batista R. O “ensino” de bioética na residência médica: a propósito de uma experiência vivenciada no Hospital Universitário Clementino Fraga Filho da Universidade Federal do Rio de Janeiro. Revista Perspectivas da Ciência e Tecnologia. 2009;1(1):22-30.

24. Kawamura K, Vicensi MC, Nodari RJ, Schlemper Junior BR, Bonamigo EL. Comitê hospitalar de bioética: êxitos e dificuldades. Rev. bioét. (Impr.). 2012;20(1):140-9.

25. Instituto Nacional de Câncer. Comunicação de notícias difíceis: compartilhando desafios na atenção à saúde. Rio de Janeiro: Inca; 2010. 
26. Universidade Federal do Rio de Janeiro. Programa de pós-graduação em bioética, ética aplicada e saúde coletiva. [Internet]. Rio de Janeiro: UFRJ. (acesso 25 set. 2013). Disponível: http://www. ppgbios.ufrj.br/

27. Vollmann J. Clinical ethics committees and ethics consultation in psychiatry. In: Helmchen $\mathrm{H}$, Sartorius N, organizadores. Ethics in psychiatry: european contributions. London: Springer; 2010.

\section{Participação dos autores}

Suely Marinho e Alexandre Costa: discussão da estrutura, elaboração do texto e revisão final - ambos integram a equipe de implantação e desenvolvimento dos comitês nos hospitais; Sergio Rego e Marisa Palácios: coordenadores gerais do projeto de extensão contribuíram também na discussão da estrutura e revisão final do texto.

Recebido: 11.10 .2013

Revisado: 19.11 .2013

Aprovado: 2. 2.2014 